\title{
Aggregate formation in a system of coagulating and fragmenting particles with mass-dependent diffusion rates
}

\author{
R. Rajesh, ${ }^{1, \text { f Dibyendu Das, }}{ }^{2}$ Bulbul Chakraborty, ${ }^{2}$ and Mustansir Barma ${ }^{3}$ \\ ${ }^{1}$ Department of Physics-Theoretical Physics, University of Oxford, 1 Keble Road, Oxford OX1 3NP, UK \\ ${ }^{2}$ Martin Fisher School of Physics, Brandeis University, 415 South Street, Waltham, MA 02454, USA \\ ${ }^{3}$ Department of Theoretical Physics, Tata Institute of Fundamental Research, Homi Bhabha Road, Mumbai-400005, India
}

(Dated: November 12, 2018)

\begin{abstract}
The effect of introducing a mass dependent diffusion rate $\sim m^{-\alpha}$ in a model of coagulation with single-particle break up is studied both analytically and numerically. The model with $\alpha=0$ is known to undergo a nonequilibrium phase transition as the mass density in the system is varied from a phase with an exponential distribution of mass to a phase with a power-law distribution of masses in addition to a single infinite aggregate. This transition is shown to be curbed, at finite densities, for all $\alpha>0$ in any dimension. However, a signature of this transition is seen in finite systems in the form of a large aggregate and the finite size scaling implications of this are characterized. The exponents characterizing the steady state probability that a randomly chosen site has mass $m$ are calculated using scaling arguments. The full probability distribution is obtained within a mean field approximation and found to compare well with the results from numerical simulations in one dimension.
\end{abstract}

PACS numbers: 64.60.-i, 05.40.-a, 61.43.Hv

\section{INTRODUCTION}

Systems far from equilibrium can undergo phase transitions between two types of steady states when the parameters of the system are varied. It is important to ask about the sensitivity of such nonequilibrium phase transitions to changes in the governing dynamics. If the transition survives, is the universality class affected? If the transition is lost, does a signature of the lost phase remain in any form?

In this paper, we investigate these questions within a lattice model of coagulation and fragmentation in which the diffusion constant for a mass $m$ varies as $m^{-\alpha}$ with $\alpha>0$. For the case in which diffusion is independent of the mass $(\alpha=0)$ and fragmentation involves only chipping off of unit masses, it is known that there is a phase transition from a low-density phase with an exponential distribution of masses to a high-density phase with a power-law distribution of masses in addition to an infinite aggregate with a mass proportional to the volume $V$ [1]. This transition is characterized by a new universality class, different from familiar classes such as directed percolation or the parity-conserving class [2], wetting transitions, roughening transitions or boundary-driven transitions [3]. We will show below that this high-density phase is lost as soon as $\alpha$ is nonzero. Remarkably, though, an imprint of the infinite aggregate remains in the form of a large aggregate that strongly modifies the finite-size behavior of the system, and we characterize the scaling implications of this.

Let us summarize the results of earlier related work. Enhancement of aggregation moves with increasing mass,

*Electronic address: r.ravindran1@physics.ox.ac.uk corresponding to negative values of $\alpha$, have been investigated earlier in the context of coalescing branched polymers. For $\alpha=-1$, using a Smoluchowski approach it was shown that the system undergoes a gelation transition, i.e., an aggregate that subsumes a finite fraction of the total mass forms at finite time. The fragmentation move was shown to modify the mass distribution power law exponent at the gelation transition [4]. An off-lattice version of the $\alpha=0$ case was studied [5] using Smoluchowski rate equations in the context of aggregation in

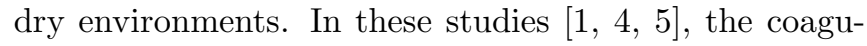
lating and fragmenting masses represented polymers in a solution, undergoing polymerization and depolymerization. In a realistic situation, it may be expected that the diffusion of the polymers would depend on their masses. For example, in the well known models of polymer motion such as the Rouse model or the Zimm model [6], the polymer diffusion constant $D(m) \sim m^{-1}$ and $m^{-1 / 2}$ respectively. This would correspond to $\alpha=1$ and $1 / 2$ in our model. This provides a further motivation for studying the model with a mass-dependent diffusion rate.

Other modifications of the dynamics of the $\alpha=0$ model that have been studied include changes of the fragmentation rule, the introduction of a spatial bias in the dynamics, and the effects of quenched disorder. Introduction of a mass-dependent fragmentation by allowing fractions of masses to break off (as opposed to singleparticle break up) was studied in [7, 8). In this case, it could be inferred that the phase transition is curbed in all dimensions. Spatial bias was introduced by choosing rates such that masses have a preferred direction of motion, but with mass-independent hopping rates. In this case, it was shown that the phase transition is curbed in one dimension [9]. In two and higher dimensions, it was shown that bias is irrelevant at least as far as the existence of a phase transition was concerned. Finally, in 
a disordered model where fragmentation of masses could occur only at fixed sites, it was shown that even in the limit of very low disorder, a new mechanism for the formation of localized infinite aggregates sets in [10].

The remainder of the paper is organized as follows. Section II contains the definition of the model, a brief review of earlier results and a summary of results obtained in this paper. Section III contains the analytical proof for the nonexistence of a phase with an infinite aggregate at large densities for any non-zero value of $\alpha$. In Sec. IV, the exponents associated with the probability distribution $P(m)$ are determined using scaling arguments. Results of Monte Carlo simulations in one dimension are also presented. In Sec. V, the full distribution is obtained from a mean field approximation and compared with the $P(m)$ obtained from numerical simulations. The appendix discusses different limiting cases of the problem that are solvable exactly.

\section{MODEL AND RESULTS}

\section{A. The model}

The model is defined on a $d$-dimensional hyper-cubic lattice with periodic boundary conditions. Starting from a random distribution of non-negative integer masses at each site, the system evolves in time via the following microscopic moves : (1) each mass $m$ hops with rate $D(m)=m^{-\alpha}$ to one of its nearest neighbor sites chosen randomly (2) with rate $w$, unit mass breaks off from an already existing mass and is transferred to a randomly chosen neighboring site and (3) following moves (1) and (2), the mass at each site adds up. The mass density $\rho$ is a conserved quantity in the model.

In one dimension, this model can be mapped [1] onto other well studied models of nonequilibrium statistical mechanics. By interpreting the masses as interparticle spacings, the model is mapped onto a one-dimensional hard core lattice gas model with competing short and long range hops. Correspondingly, the problem may be mapped onto a fluctuating interface with competing short and long range moves. The limiting case $w=\infty$ reduces to the well studied simple exclusion process 11 or equivalently to a fluctuating interface governed by the Edwards-Wilkinson equation [12].

\section{B. Previous results for $\alpha=0$}

The case $\alpha=0$ was studied by means of a mean field approximation [1], analytical calculations [13] and numerical simulations in [1, 13. The results are summarized below. The steady state single-site mass distribution $P(m)$ was shown to undergo a phase transition in all dimensions. In the $\rho-w$ plane, there is a critical line $\rho_{c}(w)=\sqrt{1+w}-1$ that separates two types of asymptotic behavior of $P(m)$. For fixed $w$, as $\rho$ is varied across the critical line $\rho_{c}(w)$, the large $m$ behavior of $P(m)$ was shown to be

$$
P(m) \sim \begin{cases}e^{-m / m^{*}}, & \rho<\rho_{c}(w), \\ m^{-\tau}, & \rho=\rho_{c}, \\ m^{-\tau}+\text { infinite aggregate, }, & \rho>\rho_{c}(w),\end{cases}
$$

where by "infinite aggregate", we mean a cluster that contains a finite fraction of the total mass in the system. That is, the tail of the mass distribution changes from an exponential decay to an algebraic one as $\rho$ approaches $\rho_{c}$ from below. As one increases $\rho$ beyond $\rho_{c}$, the asymptotic algebraic part of the critical distribution remains unchanged but in addition an infinite aggregate forms. All the additional mass in excess of the critical mass condenses into this single cluster and does not disturb the background critical distribution. The mathematical mechanism giving rise to the formation of the infinite aggregate at the onset of the phase transition was found to be very similar to that of the equilibrium Bose-Einstein condensation in an ideal Bose gas.

Finite size effects in the aggregate phase were studied in [13]. For a system of size $V$, the probability distribution $P(m, V)$ for $\rho \geq \rho_{c}$ was assumed to have the scaling form

$$
P(m, V) \approx \frac{1}{m^{\tau}} f\left(\frac{m}{V^{\chi}}\right)+\frac{1}{V} \delta\left[m-\left(\rho-\rho_{c}\right) V\right],
$$

where the exponent $\chi$ is a crossover exponent, and the $\delta$ function indicates the aggregate part. The exponents $\chi$ and $\tau$ were shown to be related by the scaling relation $\chi(\tau-1)=1$. The exponent $\tau$ was shown to be $5 / 2$ in the mean field approximation [1]; further, numerical evidence was presented [13 for the exponent being the same in all dimensions.

\section{Summary of new results in this paper}

The principal results obtained in this paper are summarized below.

(i) It is shown analytically that there is no phase transition at finite density for any $\alpha>0$ in any dimension.

(ii) On an infinite lattice with fixed density $\rho$, on assuming a scaling form

$$
P(m, \rho)=\frac{1}{m^{\tau^{\prime}}} f\left(\frac{m}{\rho^{\phi}}\right),
$$

where $f(y)$ falls exponentially as $y \rightarrow \infty$, it is shown that the two exponents are related to each other by the scaling relation

$$
\phi\left(2-\tau^{\prime}\right)=1
$$

The power law exponent $\tau^{\prime}$ is shown to be equal to

$$
\tau^{\prime}= \begin{cases}2-\frac{\alpha}{2} & \text { for } 0<\alpha \leq 2 \\ 1 & \text { for } \alpha>2\end{cases}
$$


Equivalently,

$$
\phi= \begin{cases}\frac{2}{\alpha} & \text { for } 0<\alpha \leq 2 \\ 1 & \text { for } \alpha>2\end{cases}
$$

(iii) In numerical simulations on a finite one dimensional lattice, it is seen that an aggregate forms when the total mass in the system is increased beyond a certain critical value. By analogy with the $\alpha=0$ case, we make the assumption that $P(m)$ has the scaling form,

$$
P(m, V) \approx \frac{1}{m^{\tau}} g\left(\frac{m}{V \chi}\right)+\frac{1}{V} \delta\left(m-M-M_{c}\right),
$$

where $M_{c}$ is a $V$ dependent critical mass. It is argued that $\tau^{\prime}=\tau$ with $\chi$ being related to $\tau$ through $\chi(\tau-1)=1$ as in the $\alpha=0$ case. The critical mass is shown to scale with system size as

$$
M_{c} \sim V^{2 /(2-\alpha)}, \quad \text { for } \alpha<2,
$$

implying that the critical density $\rho_{c}=M_{c} / V$ diverges with the system size.

(iv) By means of a mean field approximation, we obtain the full probability distribution $P(m)$. The scaling form Eq. (3) is seen to hold with the exponents as given in Eqs. (5) and (6).

\section{ARGUMENTS FOR NO PHASE TRANSITION AT FINITE DENSITY FOR $\alpha>0$}

On a finite lattice, on increasing the total mass $M$ from zero to large values, the following behavior is observed in numerical simulations. For small values of $M$, $P(m)$ is seen to have an exponential tail for large mass (see Fig. 1). As $M$ is increased to a critical value $M_{c}$, $P(m)$ changes to a power law with a cut off at large $m$. As $M$ is increased beyond $M_{c}$, an aggregate forms that contains all the mass in excess of $M_{c}$. The rest of the distribution remains identical to the one at $M_{c}$. The power law part has a lattice size dependent cutoff (see inset of Fig. 11). All these observations are qualitatively similar to the $\alpha=0$ case. A crucial difference is the fact that the power law exponent is seen to be less than 2.0 for $\alpha>0$. This is a puzzle since finite density would imply that $\tau>2$. In this section, we prove that there is in fact no transition at finite densities in the thermodynamic limit. The transition seen in finite size simulations is explained by the fact that $M_{c}$ no longer scales as $V$ (as in the $\alpha=0$ case), but with a power of $V$ greater than unity.

We show that an aggregate with $M_{c} \propto V$ cannot be stable at finite densities by assuming the presence of such an aggregate and showing that this leads to a contradiction. In Sec. III A, we study the mass profile as a function of distance from the aggregate. Based on our observation that at distances far from the aggregate the state of the system resembles that at the transition point, we obtain exact relations that the critical point should satisfy. In

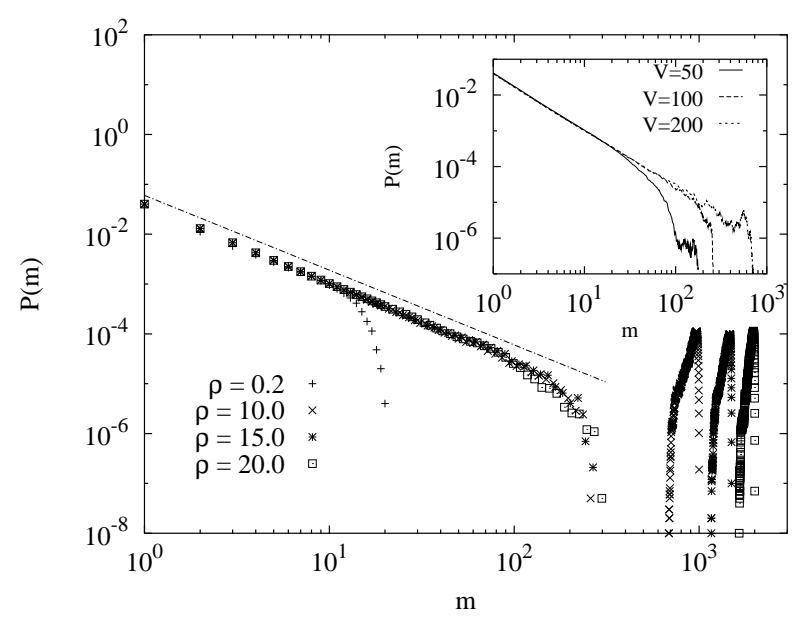

FIG. 1: The variation of $P(m)$ with $m$ for $\alpha=1.0$ is shown for four different values of density $\rho$ at fixed lattice size $V$. As density is increased, $P(m)$ changes from an exponential distribution to a power law distribution. On increasing $\rho$ further, the power law part remains unchanged while the mass in excess of a critical density $\rho_{c}$ condenses into an aggregate. The straight line has a power -1.5 . The simulation results are for a one-dimensional lattice of size 100 and $w=0.1$. In the inset, the variation of the power law cutoff with system size is shown. The simulation results are for a one-dimensional lattice with $\rho=10.0$ and $w=0.1$.

Sec. III B, we derive further exact relations by examining the two point correlations. In Sec. III , we show that the relations obtained from Secs. III A and IIIB, when put together, imply that there can be no phase transition at finite densities.

\section{A. Reference frame fixed to the aggregate}

In the aggregate phase of the $\alpha=0$ model, it is known that there exists only one large aggregate [13] in steady state; if there were more than one, they would collide and coalesce into one. This scenario is verified as well in numerical simulations for arbitrary $\alpha$ (the area under the aggregate part in the mass distribution being equal to $1 / V)$. Further, in the limit $V \rightarrow \infty$, the aggregate becomes immobile for $\alpha>0$ because its mass diverges with system size.

Consider a frame of reference that is attached to this aggregate. Let $m_{\mathbf{x}}$ and $s_{\mathbf{x}}$ denote the mass and occupation probability at a site $\mathbf{x}$ with respect to the aggregate. Then, by examining the inflow and outflow of mass at each site, we obtain

$$
\begin{aligned}
\frac{d\left\langle m_{\mathbf{x}}\right\rangle}{d t}= & -\left[w s_{\mathbf{x}}+\left\langle m_{\mathbf{x}}^{1-\alpha}\right\rangle\left(1-\delta_{\mathbf{x}, \mathbf{0}}\right)\right] \\
& +\frac{1}{2 d} \sum_{\mathbf{x}^{\prime}}\left(w s_{\mathbf{x}^{\prime}}+\left\langle m_{\mathbf{x}^{\prime}}^{1-\alpha}\right\rangle\right),
\end{aligned}
$$

with $s_{\mathbf{0}}=1$ and $\left\langle m^{y}\right\rangle=\sum_{m=1} P(m) m^{y}$. In the steady 
state, the time derivative is set to zero. Then, the solution of Eq. (9) is

$$
\left\langle m_{\mathbf{x}}^{1-\alpha}\right\rangle+w s_{\mathbf{x}}=w \quad \text { for } \mathbf{x} \neq 0 .
$$

At distances far away from the aggregate, the state of the system resembles that at criticality. Taking the limit $|\mathbf{x}| \rightarrow \infty$ in Eq. (10), we obtain

$$
\left\langle m^{1-\alpha}\right\rangle_{c}=w\left(1-s_{c}\right) \text { for } \alpha>0 .
$$

This is a relation that the system should satisfy at the critical point.

In the case $\alpha=0$, the aggregate is mobile. When the aggregate hops, this corresponds to all the other particles simultaneously making a hop with respect to the aggregate. An analysis, similar to the one carried out for $\alpha>0$, yields

$$
2 \rho_{c}=w\left(1-s_{c}\right) \quad \text { for } \alpha=0 .
$$

The origin of the factor 2 may be traced to the fact that the aggregate is mobile.

\section{B. Two point correlations}

In this section, we derive further exact relations that the system satisfies at the critical point by studying the two-point correlations. The analysis is similar to the analysis done for the $\alpha=0$ case 13 . In the rest of the paper, we will work in a coordinate system fixed to an arbitrary fixed site. To fix notation, $\mathbf{x}^{\prime}$ will always denote one of the $2 d$ nearest neighbors of $\mathbf{x}$, while $\mathbf{x}_{o}$ will denote a neighbor of the origin $\mathbf{0}$. Let $\eta\left(\mathbf{x}, \mathbf{x}^{\prime}, t\right)$ be the mass transferred from site $\mathbf{x}$ to $\mathbf{x}^{\prime}$ at time $t$ in a time interval $\Delta t$. From the definition of the model, it follows that

$$
\eta\left(\mathbf{x}, \mathbf{x}^{\prime}, t\right)= \begin{cases}m_{\mathbf{x}} & \text { with prob. } \frac{1}{2 d} \frac{\Delta t}{m_{\mathbf{x}}^{\alpha}} \\ 1-\delta_{m_{\mathbf{x}}, 0} & \text { with prob. } \frac{1}{2 d} w \Delta t \\ 0 & \text { otherwise. }\end{cases}
$$

To order $\Delta t$, the only nonzero two point correlation in the noise is

$$
\left\langle\eta\left(\mathbf{x}_{1}, \mathbf{x}_{1}^{\prime}\right)^{2}\right\rangle=\frac{\Delta t}{2 d}\left[m_{\mathbf{x}_{1}}^{2-\alpha}+w\left(1-\delta_{m_{\mathbf{x}_{1}}, 0}\right)\right] .
$$

The mass $m_{\mathbf{x}}(t)$ at lattice site $\mathbf{x}$ at time $t$ evolves as

$$
m_{\mathbf{x}}(t+\Delta t)=m_{\mathbf{x}}(t)-\sum_{\mathbf{x}^{\prime}} \eta\left(\mathbf{x}, \mathbf{x}^{\prime}, t\right)+\sum_{\mathbf{x}^{\prime}} \eta\left(\mathbf{x}^{\prime}, \mathbf{x}, t\right) .
$$

To obtain the two point correlations, we multiply $m_{\mathbf{x}}(t+$ $\Delta t)$ by $m_{\mathbf{0}}(t+\Delta t)$ and take averages over the possible stochastic moves and then over the steady state ensemble of states. Dropping all time derivatives and using Eqs. (13), (14) and (15), we obtain

$$
\begin{gathered}
C_{\alpha}(\mathbf{x})-w D(\mathbf{x})-\frac{1}{2 d} \sum_{\mathbf{x}^{\prime}}\left[C_{\alpha}\left(\mathbf{x}^{\prime}\right)-w D\left(\mathbf{x}^{\prime}\right)\right] \\
=\left[C_{\alpha}(\mathbf{0})+w s\right]\left(\delta_{\mathbf{x}, \mathbf{0}}-\frac{1}{2 d} \sum_{\mathbf{x}_{o}} \delta_{\mathbf{x}, \mathbf{x}_{o}}\right)
\end{gathered}
$$

where $C_{\alpha}(\mathbf{x})=\left\langle m_{\mathbf{x}} m_{\mathbf{0}}^{1-\alpha}\right\rangle$ and $D(\mathbf{x})=\left\langle m_{\mathbf{x}} \delta_{m_{\mathbf{0}}, 0}\right\rangle$. The homogeneous part of Eq. (16) is the Laplace equation $\nabla^{2}\left[C_{\alpha}(\mathbf{x})-w D(\mathbf{x})\right]=0$. With the inhomogeneous part, the unique solution is

$$
C_{\alpha}(\mathbf{x})=w[D(\mathbf{x})-s] \quad \text { for } \mathbf{x} \neq \mathbf{0} .
$$

Equation (17) is a relation between two point correlations. A relation between one point functions is obtained by summing over all $\mathbf{x}$, the simplifying factor being that total mass is conserved. Thus,

$$
M\left\langle m^{1-\alpha}\right\rangle-\left\langle m^{2-\alpha}\right\rangle=w M(1-s)-w s V+w s
$$

This is an exact relation in all dimensions.

We are interested in the limit when $M, V \rightarrow \infty$ keeping the density $\rho$ fixed. Taking this limit in Eq. (18), we obtain

$$
\rho\left\langle m^{1-\alpha}\right\rangle-\frac{\left\langle m^{2-\alpha}\right\rangle}{V}=w \rho(1-s)-w s, \quad V \gg 1 .
$$

In the exponential phase, $\left\langle m^{2-\alpha}\right\rangle$ is finite and hence $\left\langle m^{2-\alpha}\right\rangle / V \rightarrow 0$ as $V \rightarrow \infty$. At the transition point and in the aggregate phase $\left\langle m^{2-\alpha}\right\rangle$ can at most diverge as $V^{1-\alpha}$ (cf. discussions in the later sections of this paper). This implies that $\left\langle m^{2-\alpha}\right\rangle / V \rightarrow 0$ as $V \rightarrow \infty$ for all finite densities and any $\alpha>0$. Thus, another exact relation at the critical point is obtained:

$$
\rho_{c}\left\langle m^{1-\alpha}\right\rangle_{c}=w \rho_{c}\left(1-s_{c}\right)-w s_{c}
$$

\section{Proof of no transition}

We combine the results of Secs. III A and III B to show that there is no transition. The three quantities $\left\langle m^{1-\alpha}\right\rangle_{c}$, $\rho_{c}$ and $s_{c}$ have to simultaneously satisfy two relations, namely Eqs. (11) and (20). For non-zero values of $\alpha$, this is possible only when either $w=0$ and $\rho_{c}=0$ or $\rho_{c}=\infty$. Equations (11) and 20 cannot be satisfied at finite nonzero values of $\rho_{c}$. This completes the proof that there is no transition for $\alpha>0$ at finite critical density $\rho_{c}$.

As a check of correctness, $\rho_{c}$ and $s_{c}$ can be calculated for the $\alpha=0$ case from Eqs. (12) and (20). We obtain $\rho_{c}(w)=\sqrt{1+w}-1$ and $s_{c}=(w+2-2 \sqrt{1+w}) / w$. Not surprisingly, this is the result that had been obtained in 113 for the $\alpha=0$ case.

The fact remains that a single large aggregate is seen in simulations on a finite lattice when the mass is large enough (see Fig. 11). This observation would be consistent with the above result that there is no transition, provided the critical density $\rho_{c}$ seen in simulations diverges with $V$ as $V^{\beta}$ with $\beta>0$. We address this in the next section. 


\section{SCALING FORMS FOR PROBABILITY DISTRIBUTION $P(m, \rho, V)$}

\section{A. Large finite densities}

In this subsection, we consider the case when the total mass $M$ and the system size $V$ are increased to infinity keeping the density $\rho=M / V$ fixed. In this case, the system is always in the exponential phase. We assume the following scaling form for the probability distribution:

$$
\lim _{V \rightarrow \infty} P(m, V, \rho) \sim \frac{1}{m^{\tau^{\prime}}} f_{\alpha}\left(\frac{m}{\rho^{\phi}}\right)
$$

where $\tau^{\prime}$ and $\phi$ are two unknown exponents. Rigorous upper and lower bounds can be placed on $\tau^{\prime}$. Clearly, $\langle m\rangle=\int d m m P(m, V, \rho)$ should diverge as $\rho$ when $\rho \rightarrow$ $\infty$. But, the different moments of $m$ vary with $\rho$ as

$$
\int d m m^{y} P(m, \rho)=\int d m m^{y-\tau^{\prime}} f_{\alpha}\left(\frac{m}{\rho^{\phi}}\right) \sim \rho^{\phi\left(1+y-\tau^{\prime}\right)} .
$$

This implies that $\tau^{\prime} \leq 2$. Also, from Eq. (19), $\left\langle m^{1-\alpha}\right\rangle$ is seen to be finite for all $\rho$, in particular for $\rho \rightarrow \infty$. This implies that $\tau^{\prime}>2-\alpha$. Also, from the requirement that probability distribution sums up to $1, \tau^{\prime}$ necessarily has to be greater than 1 . These bounds can be summarized as

$$
\max (2-\alpha, 1)<\tau^{\prime} \leq 2
$$

The two exponents $\tau^{\prime}$ and $\phi$ can be expressed in terms of one another by an exponent equality. The average mass $\langle m\rangle=\rho$. This implies that

$$
\phi\left(2-\tau^{\prime}\right)=1
$$

Thus, there is only one independent exponent. $\tau^{\prime}$ is determined in Sec. IV B by studying the finite size corrections to the probability distribution.

\section{B. Aggregate formation on large finite lattices}

For a system on a finite lattice, we see (Fig. 11) that when the total mass is increased beyond a critical mass $M_{c}(V)$, the probability distribution has a $V$-dependent cutoff. Any additional mass that is added aggregates together to form one massive aggregate. Using this information, we assume the following form for the probability distribution:

$$
P(m, V)=\frac{1}{m^{\tau}} g_{\alpha}\left(\frac{m}{V^{\chi}}\right)+\frac{1}{V} \delta\left(m-\left(M-M_{c}\right)\right),
$$

where $M$ is the total mass in the system. The two exponents $\tau$ and $\chi$ can be expressed in terms of the two other exponents $\tau^{\prime}$ and $\phi$. We then determine $\tau$ by scaling arguments, thus solving for all the exponents.
In [13], it was shown that $\tau$ and $\chi$ are related by the scaling relation

$$
\chi(\tau-1)=1
$$

The derivation of this result was based on the fact that the number of aggregates is of order unity. The arguments carry forward to the general $\alpha$ case without any modification. We now argue that $\tau^{\prime}=\tau$ from Eqs. (21) and (25). The system feels the presence of the finite size when the density dependent cutoff in Eq. (21) becomes of the same order as the lattice size dependent cutoff in Eq. (25). That is, when $\rho_{c}^{\phi} \sim V^{\chi}$, or $\rho_{c} \sim V^{\chi / \phi}$. But, $\rho_{c}$ is the mean value of the mass in the power law part and from Eq. (25), $\rho_{c} \sim V^{\chi(2-\tau)}$. Thus,

$$
\chi(2-\tau)=\frac{\chi}{\phi}
$$

Substituting for $\phi$ in terms of $\tau^{\prime}$ (see Eq. (24)), we obtain

$$
\tau^{\prime}=\tau
$$

That leaves only one undetermined exponent in terms of which all the other exponents can be expressed.

To determine this exponent, we start with Eq. (18) at the transition point, namely,

$$
M_{c}\left\langle m^{1-\alpha}\right\rangle_{c}-\left\langle m^{2-\alpha}\right\rangle_{c}=w M_{c}\left(1-s_{c}\right)-w s_{c} V+w s_{c}
$$

Unlike the scaling $M / V=\rho$ that we used in deriving Eq. (19) from Eq. (18), we now assume that $M_{c}$ scales as some power of $V$, namely $M_{c} \sim V^{\beta+1}$, with $\beta>0$. From Eq. (25), we obtain

$$
\beta=\chi(2-\tau)
$$

Firstly, by substituting Eq. (25) in Eq. (18), it is easy to derive that, to leading order in $V,\left\langle m^{1-\alpha}\right\rangle_{c}=w\left(1-s_{c}\right)$. Now, to satisfy Eq. (29), there are two cases we have to consider : (A) $\left\langle m^{2-\alpha}\right\rangle_{c} \sim V$, or (B) $\left\langle m^{2-\alpha}\right\rangle_{c} \sim$ const and $\left\langle m^{1-\alpha}\right\rangle_{c}=w\left(1-s_{c}\right)-w s_{c} V^{-\beta}+\ldots$ Case $(\mathrm{A})$ requires that $\chi(3-\alpha-\tau)=1$, which when simplified implies that $\tau=2-\alpha / 2$. Case (B) requires that $\chi(3-\alpha-\tau)<0$ and $\chi(2-\alpha-\tau) \leq-\beta$ which implies that $\tau>3-\alpha$ and $\tau \geq 2-\alpha / 2$. For $\alpha \leq 1$, these bounds are in contradiction with the rigorous bounds Eq. (23). Thus for $0<\alpha<1$, only case $(\mathrm{A})$ is viable and hence $\tau=2-\alpha / 2$. For $1<\alpha \leq 2$, we have to consider case (B) also. However, any solution that arises from choosing case (B) would imply a non monotonic dependence of $\tau$ on $\alpha$. However, we expect that $\tau$ is a monotonic function of $\alpha$, and hence we discard the solutions arising from case (B). Thus, $\tau=$ $2-\alpha / 2$. For $\alpha>2$, this solution is in contradiction with the rigorous lower bound Eq. 23.). Therefore, we assume that the exponent value is stuck at 1 for all $\alpha>2$ (There is no contradiction with the above derivation since if the distribution were indeed a power law, then the integrals would now diverge at the lower cutoff too). This agrees 
with the exact solution of the $\alpha=\infty$ case (see Appendix) in which case $\tau=1$. Thus,

$$
\tau= \begin{cases}\frac{5}{2} & \text { for } \alpha=0, \\ 2-\frac{\alpha}{2} & \text { for } 0<\alpha \leq 2, \\ 1 & \text { for } \alpha>2,\end{cases}
$$

where the value for $\alpha=0$ is from [1, 13]. Solving for the other exponents, we obtain, for $0<\alpha<2$,

$$
\begin{aligned}
& \chi=\frac{2}{2-\alpha}, \\
& \beta=\frac{\alpha}{2-\alpha}, \\
& \phi=\frac{2}{\alpha} .
\end{aligned}
$$

Now that all the exponents are known, we return to the behavior of the scaling function associated with $P(m)$ at large finite densities. Numerically, we observe that the scaling function $f_{\alpha}(x) \sim$ const as $x \rightarrow 0$ for $0<$ $\alpha<2$. For $\alpha>2$, we expect $f_{\alpha}(x)$ to go to zero as some power of $x$ as $x \rightarrow 0$ (see Sec. $\mathrm{V}$ for numerics). This means that, for $\alpha<2$, in the limit $\rho \rightarrow \infty$, the probability distribution is a power law despite the mean mass diverging. These observations are consistent with the exact solution of the $\alpha=\infty$ case (see Appendix). The formation of a power law in the limit of $\rho \rightarrow \infty$ is similar to observations in models of aggregation in the presence of a constant influx of particles from outside [14, 15]. In these models, despite the mean mass diverging with time, $P(m)$ develops into a power law distribution.

An implication of the exponent $\tau$ being less than two is that the average time scale in the system may become very large. The average time scale goes as the average of the inverse of the diffusion constant, i.e. $\langle 1 / D(m)\rangle=$ $\left\langle m^{\alpha}\right\rangle \sim m_{*}^{3 \alpha / 2-1}$ where $m_{*}$ is the mass cutoff $\sim \rho^{\phi}$. Thus for $\alpha>2 / 3$, it would diverge with $m_{*}$. On the other hand, the inverse of the average diffusion constant $1 /\langle D(m)\rangle$ remain finite, since $\langle D(m)\rangle=\left\langle m^{-\alpha}\right\rangle$ is always finite. Thus our model produces a broad distribution of time scales with dissonance of average of its inverse, and inverse of its average. Such a scenario is reminiscent of diffusion in heterogeneous environment which arises in super-cooled liquids [16]. In the latter system, translational diffusion constant averaged over several heterogeneous regions falls out of proportionality with inverse of the average time scale. However, the connection of our model to the super-cooled liquids should not be taken too seriously since while the latter is in equilibrium, our model exhibits a nonequilibrium steady state.

\section{Numerical checks}

In this subsection we provide numerical support for the assertions in Sec. IVB, from Monte Carlo simulations in one dimension. Due to finite size effects, it is difficult to make an accurate direct measurement of the exponent $\tau$

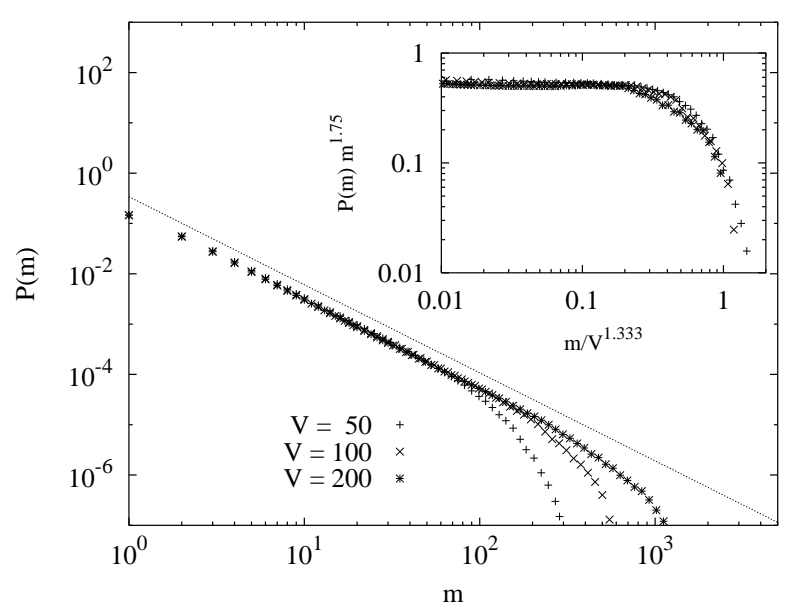

FIG. 2: The power law part of $P(m)$ obtained from Monte Carlo simulations is shown for three different values of $V$. The simulations are on a one dimensional lattice with $w=1.0$, $\rho=15.0$ and $\alpha=0.5$. The straight line has an exponent -1.75 (see Eq. (31)). In the inset the scaling plot of these curves are shown when scaled as in Eq. 25)

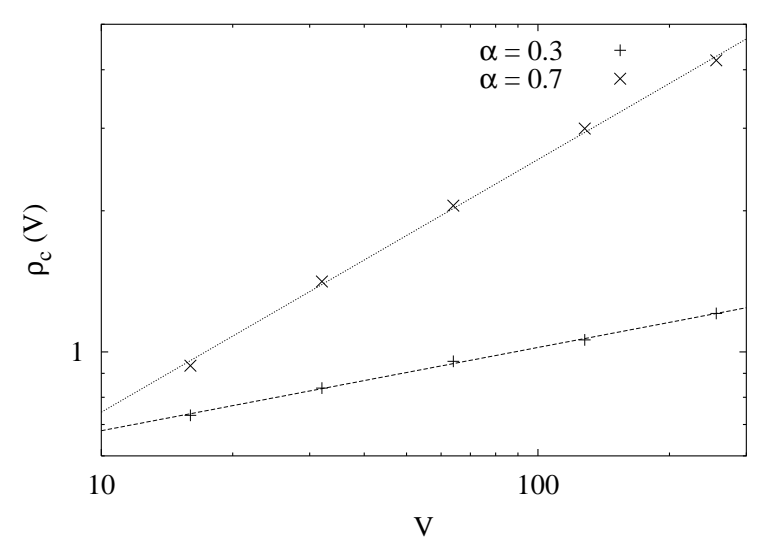

FIG. 3: The variation of $\rho_{c}(V)$ with $V$ is shown for $\alpha=$ 0.3 and $\alpha=0.7$, where the upper curve has been shifted downwards for clarity. The straight lines are best fit with power law exponents equal to $0.178 \pm 0.004$ for $\alpha=0.3$ and $0.539 \pm 0.011$ for $\alpha=0.7$. These values should be compared with the analytic results $0.176 \ldots$ for $\alpha=0.3$ and $0.538 \ldots$ for $\alpha=0.7$ (Eq. (33)). The simulation was done on onedimensional lattices for $w=1.0$.

from Monte Carlo simulations for $P(m)$. However, we show that the analytic results for the power law exponents are consistent with the numerically obtained $P(m)$. In Fig. 2, the results from simulations are compared with the analytic results for $\alpha=0.5$. In the inset, when the plots for different $V$ are scaled as in Eq. (25), the curves lie on top of each other. For $\alpha=1.0$, the predicted exponent 1.5 also matches very well with simulations (see Fig. 1).

As a second check, we measured $\rho_{c}(V)$ as a function of $V$ for $\alpha=0.3$ and $\alpha=0.7$. We adopted the follow- 
ing procedure for measuring $\rho_{c}(V)$. We start the system with total mass much greater than the critical mass $\rho_{c}(V) V$. The system is allowed to reach the steady state. The cluster with the largest mass is identified as the infinite cluster. $\rho_{c}(V)$ is obtained by measuring the average mass in the rest of the system (excluding the infinite aggregate). In Fig. 3, we obtain the exponent $\beta$ from the slope of a log-log plot of $\rho_{c}(V)$ versus $V$. There is excellent agreement with the analytically predicted values.

\section{MEAN FIELD APPROXIMATION}

In Sec. IV, the exponents characterizing the probability distribution $P(m)$ were calculated. These exponents were independent of dimension and hence should match with the mean field exponents. Also, it was observed 13] in the $\alpha=0$ case that the mean field $P(m)$ matched very well with the numerically obtained $P(m)$ for all $m$. In this section, the exponents of the probability distribution as well as the full distribution are calculated from a mean field analysis. The values of $P(m)$ thus obtained are compared with the $P(m)$ for small values of $\alpha$ obtained from Monte Carlo simulations in one dimension. From the mean field analysis, we also calculate $P(m)$ for those values of $\alpha$ which are difficult to probe by Monte Carlo simulations due to the large times required to reach the steady state.

In the mean field approximation, all correlations are ignored by setting all joint probability distributions to be the product of single point distribution functions, i.e., $P\left(m_{i}, m_{j}\right)=P\left(m_{i}\right) P\left(m_{j}\right)$. Under this approximation, the $P(m)$ 's evolve in time as

$$
\begin{aligned}
\frac{d P(m)}{d t} & =-P(m)\left(m^{-\alpha}+w+s^{\prime}+w s\right)+w P(m+1) \\
& +s w P(m-1)+\sum_{a=1}^{m} \frac{P(a) P(m-a)}{a^{\alpha}}, m>0 \\
\frac{d P_{0}}{d t} & =-s^{\prime}(1-s)-w s(1-s)+w P_{1}+s^{\prime}
\end{aligned}
$$

where $s^{\prime}=\sum_{1}^{\infty} m^{-\alpha}$. In the steady state, the time derivatives vanish. Multiplying by $e^{-p m}$ and summing $m$ from 1 to $\infty$, and eliminating $P_{1}$, we obtain

$$
Q=\frac{s Q^{\prime}+w s(1-s)\left(1-e^{-p}\right)-s s^{\prime}}{Q^{\prime}-w s-s^{\prime}-w+w e^{p}+w s e^{-p}}
$$

where $Q=\sum_{1}^{\infty} P(m) e^{-p m}, Q^{\prime}=\sum_{1}^{\infty} P(m) m^{-\alpha} e^{-p m}$ are generating functions.

The unknown quantities $s$ and $s^{\prime}$ are determined by the two conditions

$$
\begin{aligned}
\left(Q^{\prime}\right)_{p=0} & =s^{\prime} \\
\left(\frac{d Q}{d p}\right)_{p=0} & =-\rho .
\end{aligned}
$$

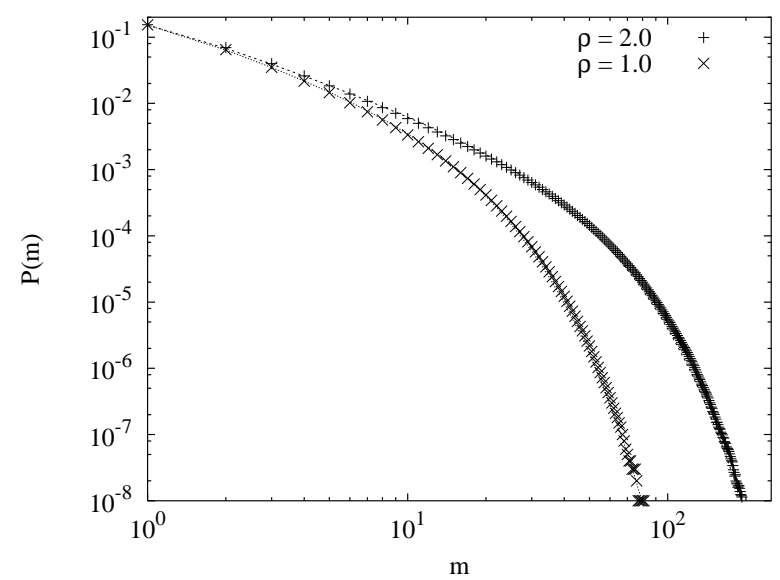

FIG. 4: $P(m)$ for $\alpha=1.0$ obtained from Monte Carlo simulations (shown in symbols) are compared with the results from the mean field analysis (shown as lines). The lattice size is $V=400$ and $w=1.0$ and we have used two densities $\rho=1.0$ and 2.0 .
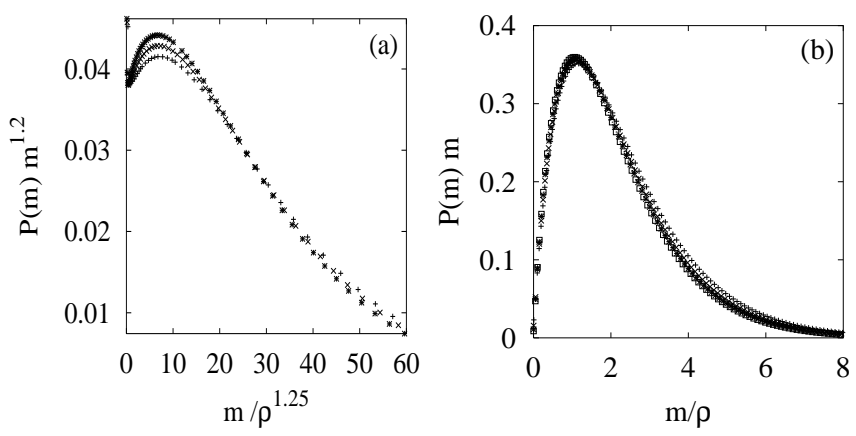

FIG. 5: $\quad P(m)$ obtained from the mean field analysis when scaled as in Eq. 21) with exponents as in Eqs. (31) and (34). (a) The curves are for $\alpha=1.6, w=0.1$ and for densities 2.38, 3.81, and 6.69. (b) The curves are for $\alpha=3.0, w=0.3$ and for densities 102.74, 165.07, 250.12 and 325.30. The scaling function $f_{3}(x)$ goes to zero for small $x$.

Using the series expansion $Q=\sum_{n=0}\left\langle m^{n}\right\rangle(-p)^{n} / n$ ! and $Q^{\prime}=\sum_{n=0}\left\langle m^{n-\alpha}\right\rangle(-p)^{n} / n !$, and comparing terms order by order in $p$, we obtain relations between moments of $P(m)$. From the term in $p^{2}$ we find

$$
\rho\left\langle m^{1-\alpha}\right\rangle=\rho w(1-s)-w s .
$$

Interestingly, Eq. (40) is identical to the exact Eq. (19) in the $V \rightarrow \infty$ limit. For $\alpha=0$ and $\alpha=1$ this yields the two results $s=\frac{\rho w-\rho^{2}}{w(1+\rho)}$ 13 and $s=\frac{\rho w}{w+\rho w+\rho}$.

Comparing the terms proportional to $p^{3}$, we obtain the relation

$$
\rho\left\langle m^{2-\alpha}\right\rangle=\left\langle m^{2}\right\rangle \frac{w s}{\rho}-\rho w(1+s) .
$$

Thus, for large $\rho,\left\langle m^{2}\right\rangle \sim \rho^{2}\left\langle m^{2-\alpha}\right\rangle$. This provides us with a method for deriving the exponents from the mean field equations. Assuming the scaling form Eq. (21) and 
using the exponent identity Eq. (24), there remain one independent exponent to calculate. Using the scaling form in $\rho$, we obtain

$$
\phi\left(3-\tau^{\prime}\right)=2+\phi\left(3-\tau^{\prime}-\alpha\right),
$$

which immediately yields $\tau^{\prime}=2-\alpha / 2$ and $\phi=2 / \alpha$, the same as in Eqs. (31) and (34).

We now calculate numerically the full $P(m)$ from the mean field Eqs. (35) and (36). If $s$ and $s^{\prime}$ are known, the full distribution $P(m)$ is known. We use this fact to determine the full distribution numerically by the following procedure. We fix $s$ and $s^{\prime}$ at a certain initial value and calculate the resulting $P(m)$ and check the consistency condition $s^{\prime}=\sum P(m) / m^{\alpha}$. We tune $s^{\prime}$ to satisfy the above condition to an accuracy of $10^{-5}$, to determine $P(m)$ 's, and thus the density $\rho$.

Using the above numerical method, $P(m)$ was calculated for various $\alpha$ 's. In Fig. 1, we compare some of these mean field results with $P(m)$ obtained using Monte-Carlo simulation, for large $V$. The agreement is excellent, suggesting that the mean field results are a very good approximation to the actual answer.

We now use the mean field results to probe $P(m)$ for values of $\alpha$ that cannot be studied easily by Monte Carlo simulations. In Fig. 5 , we show the scaling plots for $\alpha=$ 1.6 and $\alpha=3.0$. As mentioned in Sec. IVB, the small $x$ behavior of the scaling function has a different behavior for $\alpha<2$ and $\alpha>2$. In the former case $f(x) \sim$ const, while in the latter case $f(x) \sim 0$ when $x \rightarrow 0$.

\section{SUMMARY AND CONCLUSIONS}

In summary, we have studied the steady state of a system of aggregating and fragmenting particles, with a mass dependent diffusion rate $D(m) \sim m^{-\alpha}$ with $\alpha>0$. We showed analytically that the nonequilibrium phase transition which is known to exist for $\alpha=0$, vanishes when $\alpha>0$. This is in agreement with the results of extensive numerical simulations, through which we explored the dependence both on system size and total mass. Although no true infinite aggregate forms in the thermodynamic limit, its imprint at high densities remains in finite-sized systems in the form of an aggregate. Further, for the single site mass distribution function, we obtained the exact scaling exponents associated with its dependence on the mass, the density and the system size.

Our results give more credibility to the intuitive arguments presented in [9] as to the circumstances in which one should expect to see a nonequilibrium phase with an infinite aggregate, as occurs in the $\alpha=0$ case. We reproduce the argument here. In the model under consideration, there are two competing processes: while the diffusion move creates larger and larger masses by coagulation, the fragmentation move tends to create smaller masses, as well as to inhibit the formation of large masses. If the diffusion move was to be considered by itself, then a cluster of size $l$ would be created in time of order $l^{2+\alpha}$.
If the fragmentation move was to be considered on its own, then a fluctuation of order $l$ would be dissipated in time of the order $l^{2}$. This exponent is known exactly because of the exact analogy [1] in one-dimension between an only-fragmentation model and the EdwardsWilkinson interface [12]. For $\alpha=0$, the two processes are of similar strength and hence there is the possibility of a transition. But for $\alpha>0$, the fragmentation process always dominates and hence there is no aggregate phase.

\section{Acknowledgments}

We would like to thank S. Coppersmith, S. Redner, P. L. Krapivsky, D. Dhar, T. Witten and S. Krishnamurthy for helpful discussions, and especially A. Bray for his insightful suggestions on one of the limiting cases. DD and BC were supported by NSF DMR-9815986. RR would like to thank EPSRC, UK for financial support.

\section{APPENDIX A: EXACTLY SOLVABLE LIMITS}

In this appendix, we discuss the limiting cases of the model for which the full probability distribution $P(m)$ can be calculated.

$$
\text { 1. } \alpha=\infty
$$

In the limit $\alpha \rightarrow \infty$, the rate of diffusion becomes equal to zero for all masses $m \geq 2$. The model then reduces to a zero range process [17] in which with rate $w$ unit mass can break off from masses $m \geq 2$, while the unit mass can hop to a neighboring site with rate $1+w$. It is then straightforward to verify that the steady state probability distribution has a product form, i.e.,

$$
P\left(\ldots, m_{1}, m_{2}, \ldots\right)=\prod_{i} P\left(m_{i}\right),
$$

with

$$
P(m)= \begin{cases}c \gamma^{m} & m \geq 1, \\ \frac{c(1+w)}{w} & m=0 .\end{cases}
$$

The constants $c$ and $\gamma$ are fixed by the two constraints $\sum_{m} P(m)=1$ and $\sum_{m} m P(m)=\rho$. Solving for $c$ and $\gamma$, we obtain

$$
\begin{aligned}
c & =\frac{w(1-s)}{1+w}, \\
\gamma & =\frac{s(w+1)}{w+s}
\end{aligned}
$$

with the site occupation probability $s$ being equal to

$$
s=\frac{\sqrt{w^{2}(1+\rho)^{2}+4 \rho w}-w(1+\rho)}{2} .
$$


In the limit $w \rightarrow \infty, s$ has the correct limit $\rho /(1+\rho)$ (see Eq. (A9)).

We would be interested in the form of $P(m, \rho)$ when $\rho \rightarrow \infty$. Expanding $s$ in terms of $1 / \rho$, we obtain,

$$
s=1-\frac{1+w}{w} \frac{1}{\rho}+O\left(\frac{1}{\rho^{2}}\right) .
$$

In this limit

$$
P(m, \rho) \approx \frac{1}{\rho} e^{-m / \rho}, \quad \rho \rightarrow \infty .
$$

Thus

$$
P(m, \rho)=\frac{1}{m} f_{\infty}\left(\frac{m}{\rho}\right), \quad m, \rho \rightarrow \infty,
$$

where the scaling function $f_{\infty}(x) \sim x$ when $x \rightarrow 0$. From Eq. (A8), we see that $\tau=1$ for $\alpha=\infty$.

$$
\text { 2. } w=\infty
$$

In the limit $w=\infty$, the model reduces to a zero range process 17. As in the $\alpha=\infty$ case, the steady state prob- ability distribution has a product form as in Eq. A1). $P(m)$ for this limiting case was worked out in [1]. For the sake of completeness, we reproduce the final result,

$$
P(m)=\frac{1}{1+\rho}\left(\frac{\rho}{1+\rho}\right)^{m}, \quad m \geq 0 .
$$

In this limit, masses diffuse and coagulate on contact. Clearly, the steady state is one in which the entire mass is clumped together into one aggregate. For the $\alpha>$ 0 problem, this is the only limit in which an aggregate forms which holds a finite fraction (here unity) of the total mass at finite density.

[10] K. Jain and M. Barma, Phys. Rev. E 64, 016107 (2001).

[11] B. Derrida and M. R. Evans in Nonequilibrium Statistical Mechanics in One Dimension edited by V. Privman (Cambridge University Press, UK, 1997).

[12] A. -L. Barabási and H. E. Stanley, Fractal Concepts in surface growth (Cambridge University Press, 1995).

[13] R. Rajesh and S. N. Majumdar, Phys. Rev. E. 63, 036114 (2001).

[14] H. Takayasu, I. Nishikawa, and H. Tasaki, Phys. Rev. A 37, 3110 (1988).

[15] P. L. Krapivsky, J. F. F. Mendes, and S. Redner, Phys. Rev. B 59, 15950 (1999).

[16] G. Tarjus and D. Kivelson, J. Chem. Phys. 103, 3071 (1995).

[17] F. Spitzer, Adv. in Math. 5, 246 (1970); M. R. Evans, Braz. J. Phys. 30, 42 (2000). 